\title{
El uso del enfoque del estudio de caso: Una revisión de la literatura
}

\author{
The use of the case study approach: A review of the literature \\ 0 uso da abordagem do estudo de caso: uma revisão da literatura
}

ARTÍCULO DE INVESTIGACIÓN

\author{
Jorge Luis Canta Honores \\ jorge.canta@upn.pe \\ https://orcid.org/0000-0002-9232-1359
}

Universidad Privada del Norte, Trujillo, Perú
Julio Quesada Llanto

julio.quesada@upn.edu.pe

https://orcid.org/0000-0003-4366-4926

Universidad Privada del Norte, Trujillo, Perú

Recibido 27 de abril 2021 | Arbitrado y aceptado 21 de mayo 2021 | Publicado en 01 julio 2021

\section{RESUMEN}

La investigación cualitativa ha ganado mucho terreno en la actualidad, se considera legítima dentro de disciplinas donde generalmente prevalece la hegemonía del paradigma positivista cuantitativo. En ese sentido, el objeto es la revisión, análisis y reflexión acerca del uso del método de estudio de caso, destacándolo como un enfoque importante en la investigación cualitativa para obtener conocimiento significativo. En base a lo descrito, se estableció como componentes, (i) el problema de investigación y su significancia, donde se analiza las dificultades que soslaya; (ii) el marco teórico, así como criterios de selección de casos; (iii) el método de revisión de la literatura; (iv) resultados principales; (v) discusión; y (vi) conclusiones. El método aplicado para la recopilación y ordenamiento de la información de las fuentes fue la técnica de la revisión sistemática, que se utilizó para reducir los sesgos, obteniéndose resultados confiables que permitieron emitir conclusiones y decisiones. Entre los principales resultados se determinó las ventajas de la aplicación, limitaciones relevantes del estudio, las contraindicaciones del método, la validez del método como investigación cualitativa, contribuciones al conocimiento, lineamientos de aplicación de consideraciones fundamentales, viabilidad de la aplicación de los instrumentos cuantitativos en el estudio de caso y la determinación de las principales referencias incluidas. Se concluyó que el método de estudio de caso se puede aplicar a un caso (objeto de estudio) o a una muestra reducida de casos y que a partir de ello no se infiere resultados para otros estudios, con diferente ubicación y realidad social.

Palabras clave: Enfoque cualitativo; Investigación de estudio de caso; Consideraciones del estudio de caso; Ventajas del estudio de caso; Aplicaciones del estudio de caso

\section{ABSTRACT}

Qualitative research has gained much ground today, it is considered legitimate within disciplines where the hegemony of the quantitative positivist paradigm generally prevails. In this sense, the object is the review, analysis and reflection on the use of the case study method, highlighting it as an important approach in qualitative research to obtain meaningful knowledge. Based on what has been described, it was established as components: (i) the research problem and its significance, where the difficulties it avoids are analyzed; (ii) the theoretical framework, as well as case selection criteria; (iii) the method of reviewing the literature; (iv) main results; (v) discussion; and (vi) conclusions. The method applied for the collection and ordering of the information from the sources was the systematic review technique, which was used to reduce bias, obtaining reliable results that allowed conclusions and decisions to be made. Among the main results, the advantages of the application, relevant limitations of the study, the contraindications of the method, the validity of the method as a qualitative research, contributions to knowledge, application guidelines of fundamental considerations, feasibility of the application of quantitative instruments in the case study and the determination of the main references included. It was concluded that the case study method can be applied to a case (object of study) or to a reduced sample of cases and that results are not inferred from this for other studies, with different location and social reality.

Key words: Qualitative approach, Case study research, Case study considerations, Case study advantages, Case study applications

\section{RESUMO}

A pesquisa qualitativa ganhou muito terreno hoje em dia, é considerada legítima dentro de disciplinas onde geralmente prevalece a hegemonia do paradigma quantitativo positivista. Neste sentido, o objeto é a revisão, análise e reflexão sobre o uso do método de estudo de caso, destacando-o como uma abordagem importante na pesquisa qualitativa para a obtenção de conhecimentos significativos. Com base no que foi descrito, foram estabelecidos os seguintes componentes: (i) o problema de pesquisa e sua importância, onde são analisadas as dificuldades que ele ignora; (ii) a estrutura teórica, bem como os critérios de seleção de casos; (iii) o método de revisão de literatura; (iv) os principais resultados; (v) a discussão; e (vi) as conclusões. O método aplicado para a coleta e ordenação de informações das fontes foi a técnica de revisão sistemática, que foi utilizada para reduzir os enviesamentos, obtendo resultados confiáveis que permitiram tirar conclusões e tomar decisões. Entre os principais resultados foram determinadas as vantagens da aplicação, as limitações relevantes do estudo, as contraindicações do método, a validade do método como pesquisa qualitativa, contribuições ao conhecimento, diretrizes para a aplicação de considerações fundamentais, viabilidade da aplicação de instrumentos quantitativos no estudo de caso e a determinação das principais referências incluídas. Concluiu-se que o método de estudo de caso pode ser aplicado a um caso (objeto de estudo) ou a uma amostra reduzida de casos e que a partir dele não se inferem resultados para outros estudos, com localização e realidade social diferentes.

Palavras-chave: Abordagem qualitativa; Pesquisa de estudo de caso; Considerações de estudo de caso; Vantagens do estudo de caso; Aplicações do estudo de caso 


\section{INTRODUCCIÓN}

La investigación es la base del conocimiento y como tal ha sido materia de análisis incesante en los métodos que emplea, estableciéndose diversos enfoques de acuerdo a la particularidad de los objetivos de la investigación, en ese sentido, en la actualidad los enfoques de investigación de todas las ramas académicas están explícitamente delimitadas, entre el uso de una investigación cuantitativa, cualitativa o mixta. Dentro de las investigaciones cualitativas, específicamente para el método de estudio de caso, su aplicación podría allanarse a muchas interrogantes, por ejemplo, $¿ E l$ estudio de caso de un edificio, será una investigación factible para evaluar y determinar el comportamiento de las diversas edificaciones?, $¿ E l$ estudio de caso de la enfermedad de un paciente, es relevante dentro del contexto del tratamiento de una epidemia?, las interrogantes tienen que ser resueltas objetivamente en un entorno del avance de la aplicación de diseños y procedimientos, en la investigación del estudio de caso (Bruns, 1989).

El artículo expone un análisis resumido de la validez del enfoque cualitativo del estudio de caso, con la propuesta de la siguiente interrogante para el desarrollo del estudio: ¿El método de estudio de caso es relevante para la obtención de conocimiento significativo? En ese sentido, se ha recurrido a fuentes de diversas ramas de la investigación social, psicológica y médica, entre otras, indagando para tal fin, la aplicación preferente y la muestra más idónea. Se debe enfatizar que el objeto del estudio de caso, como enfoque cualitativo, es especialmente entender un fenómeno y/o explorar temas donde los conocimientos son limitados, para tal efecto, los resultados del estudio pueden acrecentar la teoría existente y conducir al investigador a etapas posteriores de la búsqueda del conocimiento (DeVaney, Spangler, Lee, y Delgadillo, 2018).

En el análisis de la problemática del estudio de caso, la principal dificultad que se presenta, se refiere esencialmente a la carencia de rigor metodológico, ante ello se ha propuesto por diversos autores, modelos explicativos a fin de mitigar dicha problemática (Creswell y Poth, 2018). Por el contrario, Smith (2018) destaca las fortalezas del estudio de caso, concluyendo que son investigaciones que cuentan con abundantes datos, que implican diversas fuentes de evidencia. Del mismo modo, Smith (2018) manifiesta que el estudio de caso es un diseño de investigación conocido de las ciencias sociales a pesar de los recelos acerca de su fiabilidad.

Los estudios de caso abarcan mucha más complejidad que un informe de caso típico, ya que incorporan múltiples flujos de datos combinados de manera creativa, en tal sentido, el detalle y riqueza, es que ayudan a los lectores a comprenderlo, así como a determinar si los hallazgos podrían ser aplicables más allá de ese entorno (Alpi y Evans, 2019). Del mismo modo, el método de estudio de caso se da a conocer como: Una investigación empírica apropiada para determinar el "cómo y por qué" de los fenómenos, asimismo contribuye a comprender los fenómenos en un contexto holístico y de la vida real, del mismo modo se conoce el caso como una cosa, una sola entidad, una unidad alrededor de la cual existen límites (Alpi y Evans, 2019, p. 2).

En la aplicación del método de estudio de caso, Alpi y Evans (2019) manifiestan que se pueden emplear una variedad de enfoques metodológicos para explicar la complejidad del problema que se está estudiando, partiendo de un enfoque cualitativo en el que se explora un sistema delimitado y contemporáneo, así como a través de una recopilación de datos detallada, que involucra múltiples fuentes de información.

En el marco teórico y conceptos relevantes, se plantea que el estudio de caso según Creswell (2018), "Es un diseño de investigación que se encuentra en muchos campos, especialmente la evaluación, en el que el investigador desarrolla un análisis en 
profundidad de un caso, a menudo un programa, evento, actividad, proceso o una o más personas" (p. 30). En el mismo sentido, la selección del caso se desarrollará en un periodo finito de tiempo, así como en una determinada área geográfica (Stake, 2005). Por otro lado, Eisenhardt, Graebner, y Sonenshein (2016) manifiestan que el desarrollo de teorías derivadas de casos, conjugan estudios de casos y razonamiento de teoría fundamentada, entendiéndose para estos estudios, que la lógica de replicación considera a cada caso como una entidad independiente. En suma, un estudio de caso se desarrolla en un escenario real y actual, siendo el objeto de estudio un ente individual, grupo o estructura organizacional, un proyecto, una comunidad o incluso un proceso de decisión (Yin, 2014).

Entre las principales aplicaciones se tiene las investigaciones sobre identidades multiculturales (Goodyear et al., 2016). Asimismo, el estudio de caso es apropiado cuando el fenómeno de interés es continuo, en lugar de histórico y no puede separarse del contexto en el que ocurre (Gupta, Forlini, Lenton, Duchen, y Lohfeld, 2016). Por otro lado, de acuerdo con Lee y Catling (2017), el uso de estudios de caso correctamente seleccionados por los investigadores, permite ser más cautelosos sobre la generalización de estos, considerando que esta no siempre es posible y que frecuentemente resulta inapropiada. En el mismo sentido, Lee y Catling (2017) sostienen que, si un estudio no contiene objetivos claros, no generará impacto, por el contrario, ocasionará impresiones negativas. Asimismo, los autores precisan que: Entre los criterios para seleccionar y desarrollar estudios de casos se tiene el conocimiento o experiencia personal del investigador, así como la importancia, se considera que el conocimiento personal de los autores, a través de la investigación y/o experiencia es una fuente valiosa para el desarrollo de estudios de caso (Lee y Catling, 2017, p. 346).
El método de caso tiene diversos usos, entre ellos destacamos su empleo como una metodología central para los académicos que trabajan en la retórica de la ciencia y la comunicación técnica (Moriarty et al., 2019). El empleo se extiende incluso como un método micro analítico para estudios de casos únicos basados en teoría en psicoanálisis (Kivikkokangas y Leiman, 2019). La investigación cualitativa desde la perspectiva del método de caso abarca mucha más complejidad que un informe de caso típico y a menudo, incorpora múltiples flujos de datos combinados de manera creativa (Alpi y Evans, 2019). Por otro lado, según Barbosa, Franz, Munteanu, y Baecker (2017), "El análisis temático se basa en un enfoque mixto (inductivo y deductivo), es decir, los temas "surgieron" de los datos, pero también de temas a priori relacionados con la adopción de tecnología (por ejemplo, utilidad)".

En el método de caso, los temas de estudio se eligen o son elegidos por los propios autores de la investigación, al respecto los autores indican: Los autores reflexionan sobre cómo los retóricos de los investigadores de la ciencia y la comunicación técnica, pueden construir una variedad de estudios de métodos mixtos basados en casos, de manera que puedan hacer que la investigación sea más portátil y duradera, del mismo modo se plantean preguntas importantes para los retóricos de la ciencia sobre el uso de la investigación de estudios de casos, destacando la selección de casos, la representatividad y la generalización como preocupaciones para la portabilidad de esta metodología (Moriarty, et al., 2019, p. 342).

El estudio de caso emplea procedimientos relevantes para llegar al conocimiento, en tal sentido Avolio (2015), sostiene que para el análisis e interpretación de datos es fundamental organizar, agrupar y explicar la información recolectada, que se torna en procesos enmarañados, confusos, que demandan demasiado tiempo, pero que a la vez son agradables e ingeniosos. Del mismo modo la autora 
manifiesta que en el enfoque cualitativo del estudio de caso, el proceso de interpretación se fundamenta en conceder significado a la información, en tal sentido la información que no cuenta con un análisis de cada caso o análisis transversal de casos, no tiene significado inherente, es decir la interpretación le confiere significado a los datos y transmite dicho significado al lector mediante un reporte escrito. En suma, Avolio (2015) plantea, "Lo primero que debe presentarse es la estrategia general utilizada para analizar los datos, como marco general. Para poder conocer las diferentes estrategias, se requiere estudiar los diversos métodos, en función del método elegido" (pp. 105-106).

\section{MÉTODO}

Se utilizó como recursos las bases de datos de referencias bibliográficas que brinda la Pontificia Universidad Católica del Perú (PUCP), tales como Scopus (SJR Quartile), Web of Science y EBSCO. Asimismo, en la PUCP se contó con la línea de apoyo de servicios DOCIS, la cual facilitó el acceso y asesoría para la obtención de información, adicionalmente se utilizó repositorios de bases abiertas como el Google Académico. La recopilación de la información sirvió para obtener un producto basado preferentemente en información de revistas científicas validadas por Scimago Institutions Rankings. El criterio de inclusión de artículos consistió en fuentes bibliográficas confiables, preponderadamente research papers (journal papers), incorporadas por Scimago Institutions Rankings, entre los Quartiles 1 y 2, publicados en el idioma inglés, con el periodo de publicación desde el año 2015 al 2019.

Las palabras clave utilizadas fueron: "enfoque cualitativo", "investigación de estudio de caso", "consideraciones del estudio de caso", "ventajas del estudio de caso" y "aplicaciones del estudio de caso". Para la elección de las palabras se consideró los aspectos principales abordados en la investigación, tales como el enfoque de investigación "enfoque cualitativo", método "investigación de estudio de caso", aspectos a tener en cuenta "consideraciones de estudio de caso", identificación de sus utilidades "ventajas del estudio de caso" y usos del método "aplicaciones del estudio de caso".

Para la recopilación y ordenamiento de la información de las fuentes se empleó la técnica de la revisión sistemática, seleccionando información relevante en base al instrumento denominado matriz de revisión sistemática, cuya estructura incorpora registros, que permiten el escudriñamiento de un artículo de investigación, en ese sentido Munn, Peters, Stern, y Tufanaru (2018) manifiestan que las revisiones sistemáticas se refieren a un tipo de síntesis de estudios aplicado por expertos. Del mismo modo una revisión sistemática utiliza métodos claros y organizados que se eligen con el objeto de reducir las desviaciones, generándose resultados confiables que permiten emitir conclusiones y decisiones.

La selección partió de los rankings de las revistas científicas consultadas, entre los Quartiles 1 y 2. En la primera revisión se seleccionó 40 research articles, reduciéndose dicha cantidad a 21 research articles, después de la aplicación de la técnica de revisión sistemática, para la versión final del artículo.

\section{RESULTADOS Y DISCUSIÓN}

Como resultado de la revisión de la literatura, se determinó en primer lugar las ventajas que ofrece el método de estudio de caso, se destaca su versatilidad, así como la estructura que aporta para explorar una variedad de problemas, lo que lo convierte en un método apropiado para iniciar un estudio exploratorio. Del mismo modo, para Ling, Payne, Connaire, y McCarrons (2015) ofrece flexibilidad para seleccionar el perfil del caso, mientras que el análisis retórico equilibra la flexibilidad al imponer un paradigma de investigación duradero (Moriarty et al., 2019). Por otro lado, la investigación por el 
método de estudio de caso busca identificar el atractivo en disciplinas aliadas y su relación con el marco teórico, asimismo interpreta información a través de múltiples formas de conocimiento cualitativo (constructivista, interpretativo, artístico, performativo), así como por medio del rol del investigador en el proceso de investigación (Leonard y Odutola, 2016). En síntesis, se propone una sucinta discusión sobre el método de estudio de caso en el marco del enfoque cualitativo, así como del resumen de sus bases teóricas, conceptos y aplicabilidad.

En segunda instancia, se estableció la limitación más relevante del estudio de caso como método de investigación, la cual se basa en el hecho de que analiza un solo objeto o grupo de estudio (Leonard y Odutola, 2016). Del mismo modo, se presenta un potencial de sesgo en la selección del caso, así como otras limitaciones del enfoque (Morgenstern, 2018). En el mismo sentido, otra condicionante de desviaciones del estudio, es que, al incluir definiciones del propio investigador, así como de la delimitación del alcance del caso o casos que se estudia, determinaría que no es generalizable para muestras mayores, sin embargo, la investigación por estudio de caso, como metodología cualitativa, busca el desarrollo de diferentes propósitos para la generalización en los diversos campos de estudio (Alpi y Evans, 2019).

Como tercer punto, entre las contraindicaciones del método de estudio de caso se tiene a los desafíos éticos debido a la naturaleza etnográfica de los datos, para lo cual se sugiere garantizar que la identidad y la privacidad de los participantes del estudio esté cubierta, indicándose el uso de principios éticos en la recopilación, el análisis y el almacenamiento de los datos (Cvitanovic, Cunningham, Dowd, Howden, y Putten, 2017). Del mismo modo, otra limitación relevante está relacionada con la metodología utilizada para la recopilación de datos para los estudios de caso, incluye la auto información de las personas involucradas, por lo tanto, los impactos representan las perspectivas individuales de los autores y requieren un análisis adicional para evaluar su consistencia en una variedad de perspectivas (Wyborn et al., 2018).

En cuarto lugar, se determinó la validez del método de estudio de caso dentro de la investigación cualitativa, que se sostiene en que el método de caso es un análisis adoptado en los estudios de una variedad de investigadores, cuyas pautas son aceptadas en parte por la literatura de investigación cualitativa (Gupta, Forlini, Lenton, Duchen, y Lohfeld, 2016). En ese sentido, de acuerdo a lo destacado por Alpi y Evans (2019), los estudios de caso contienen, preguntas y proposiciones del estudio, la unidad de análisis, la lógica que vincula los datos a las proposiciones, y los criterios para interpretar los resultados. Asimismo, los autores dan a conocer múltiples fuentes de datos, bases de datos y una cadena clara de evidencia entre las preguntas formuladas, los datos recopilados y las conclusiones extraídas.

Como quinto punto, se enfatiza que los estudios de caso agregan contribuciones al conocimiento, en ese sentido Avolio (2015) sostiene el aporte de los mismos, destacando que las contribuciones se desagregan en dos: las teóricas y las prácticas. Las teóricas están referidas al aporte de la investigación al conocimiento y teoría existente. Las prácticas se refieren al aporte de la investigación en las ciencias sociales, dependiendo de la naturaleza y propósito del estudio. Pueden darse ambas contribuciones o también solo una de ellas (Figura 1). Del mismo modo la autora propone: En los enfoques cualitativos, el proceso de interpretación es el que otorga significado a la información, ya que la información sin analizar no tiene un significado inherente, la interpretación brinda significado a los datos y los expone al lector a través del reporte escrito, lo primero que debe presentarse es la estrategia general utilizada para analizar los datos, 
como marco general, se debe tener en cuenta la forma en que se analizará la información y cuáles serán las fases y perspectivas del análisis de cada caso y del análisis transversal de casos, de no ser así el estudio no tendrá una buena calidad (p. 106).

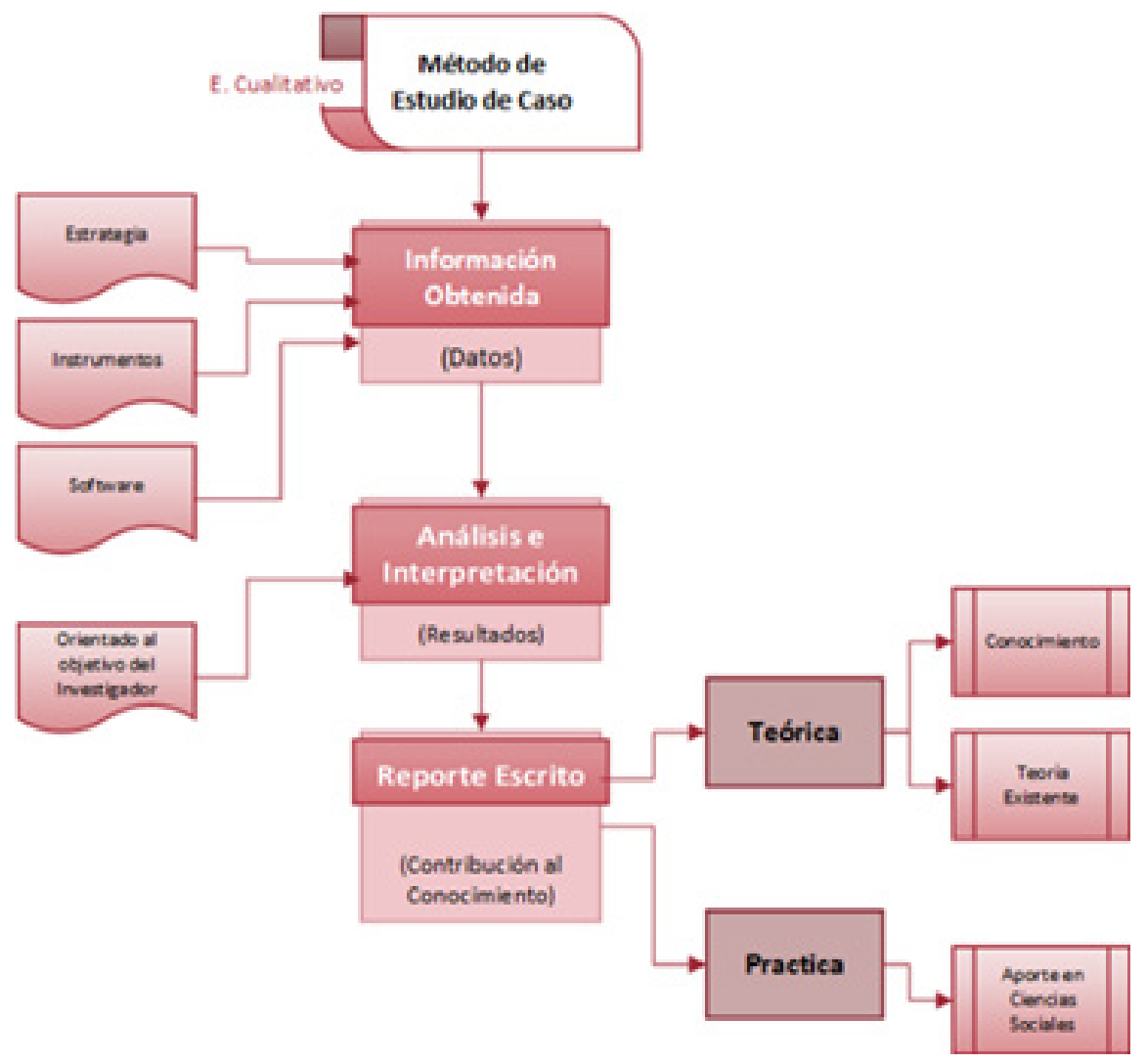

Figura 1. Adaptado de Métodos cualitativos de investigación : una aplicación al estudio de caso. (Avolio, 2015).Recuperado de http://www.ebooks7-24.com.ezproxybib.pucp.edu.pe:2048/?il=2133

En sexto lugar, para iniciar una investigación por el método de estudio de caso, se debe aplicar las consideraciones fundamentales, lo cual significa la selección de un caso o casos para para explicar el problema, describiendo al por menor su estructura (Creswell y Poth, 2018). Entre las consideraciones fundamentales se tiene, (a) el enfoque del estudio de caso, el cual es apropiado para un problema de investigación, cuando el investigador posee casos visiblemente reconocibles, así como cuando se persigue un entendimiento amplio del caso o múltiples casos (Creswell y Poth, 2018), otro aspecto para determinar el enfoque es desarrollar la pregunta de investigación (Tetnowski, 2015); (b) la unidad de análisis, al respecto Smith (2018) propone que los estudios de caso facilitan una búsqueda a fondo de la unidad de análisis; (c) el tipo de problema de investigación apto para el enfoque, para tal efecto Stake (2005) señala que el objetivo del estudio de caso es el entendimiento un problema o preocupación particular, así como la adecuada elección de un caso o casos para interpretar el problema; y (d) la naturaleza de origen disciplinario del estudio de caso, sobre ello Creswell y Poth (2018) manifiestan que este enfoque conocido para los investigadores sociales por su divulgación en la psicología, medicina, derecho y ciencias políticas (Figura 2). 


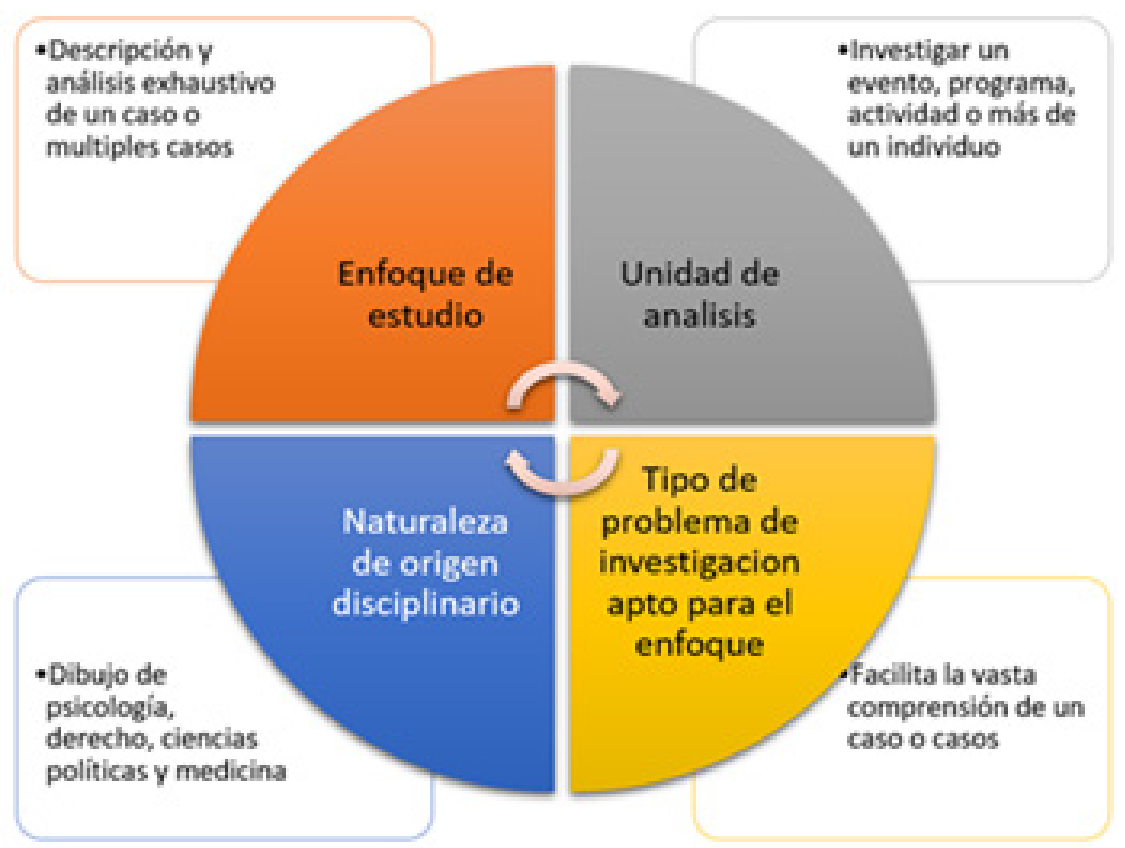

Figura 2. Consideraciones fundamentales del estudio de caso. Adaptado de Investigación cualitativa y diseño de investigación: elegir entre cinco enfoques. (Creswell y Poth, 2018).

Como séptimo punto, se determinó que es viable la aplicabilidad de los instrumentos cuantitativos en el método de estudio de caso, en tal sentido, una síntesis de investigación cualitativa ayuda a plasmar los resultados de las revisiones cuantitativas, con un énfasis emergente en los enfoques individualizados, ello permite crear una lista de recomendaciones (Flemming, Closs, Hughes, y Bennett, 2016).
Asimismo, es preciso señalar que los investigadores de estudios de caso pueden recoger datos, tanto cuantitativos, como cualitativos (Goodyear et al., 2016). En suma, es relevante que los autores sigan un proceso adecuado para la obtención datos, por ejemplo, el desarrollo exhaustivo de un estudio piloto respaldado con sus fuentes. (Figura 3).

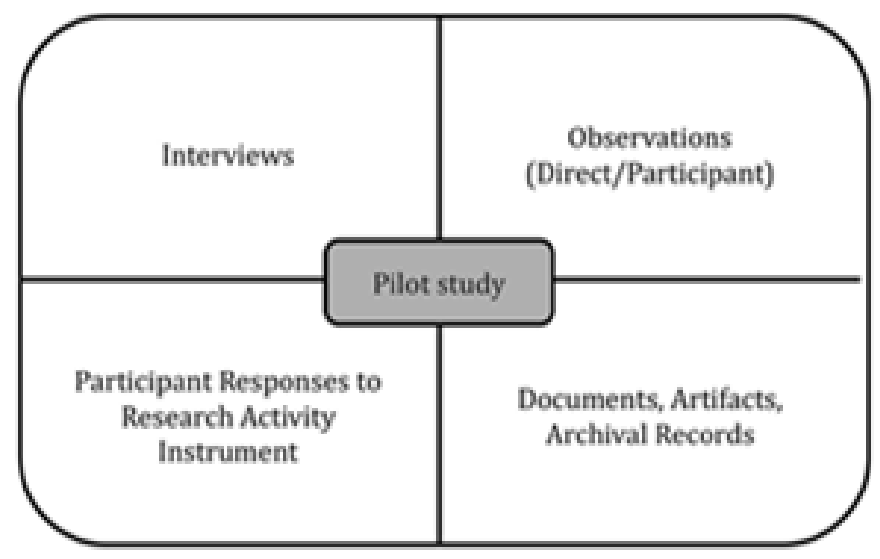

Figura 3. Fuentes clave de datos para un estudio de caso. Tomado de Distinguishing case study as a research method from case reports as a publication type. (Alpi y Evans, 2019, p. 3). 
En un octavo resultado, se muestra el detalle de las principales referencias incluidas, producto de la aplicación de la técnica de revisión sistemática, identificándose en primer lugar, 40 artículos de diversos países, idiomas y año de publicación, de los cuales se seleccionó 21, publicados desde el año 2015 al 2020, alineados a los contenidos de la investigación, preferentemente en idioma inglés de los Estados Unidos, Reino Unido, Holanda y Alemania. Del mismo modo, la mayor proporción de los 21 research papers seleccionados, es decir 17, están incorporadas por Scimago Institutions Rankings en los Quartiles 1 y 2. (Figura 4).

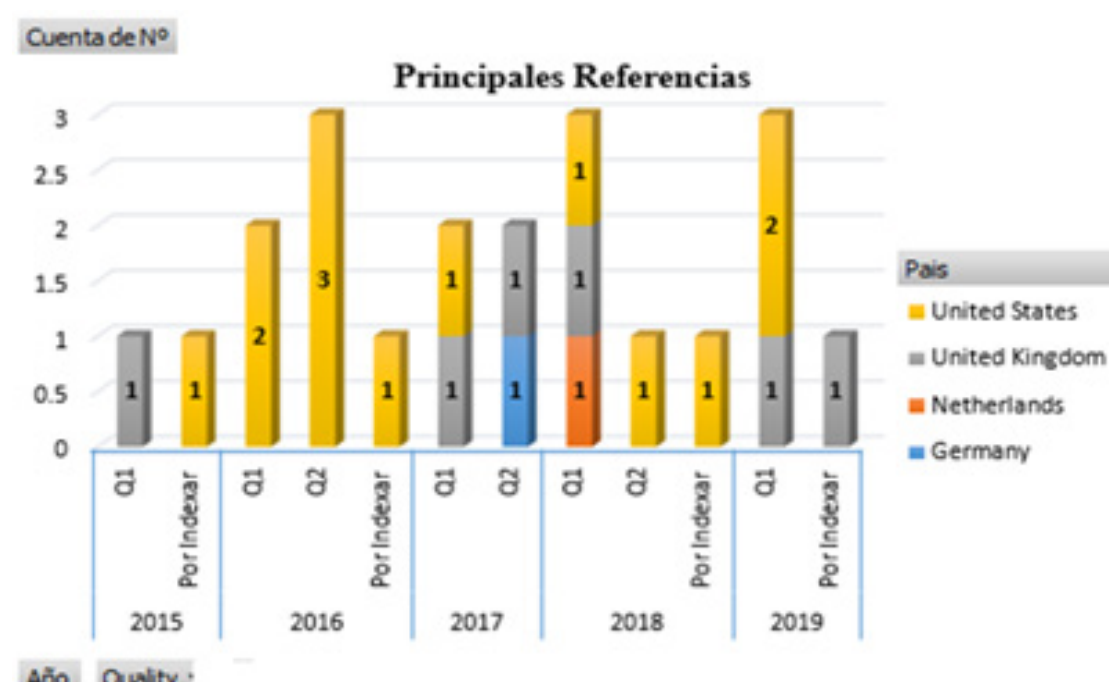

Figura 4. Análisis de artículos seleccionados.

\section{Discusión}

La validez de la aplicación de los principales enfoques cualitativos es materia de frecuente discusión en la literatura internacional, siendo el método de estudio de caso, a pesar de sus detractores, uno de los más empleados y conocidos. En tal sentido, es prioritario para la elaboración de un estudio de caso, definir el problema de investigación que se busca solucionar o mitigar. En tal sentido, un aspecto neurálgico para iniciar un estudio de caso, es tomar en cuenta las consideraciones fundamentales de este tipo de estudio, tanto en la exploración o en el incremento del conocimiento de un hecho existente o inédito. Es relevante señalar que el enfoque del estudio ha evolucionado en los últimos años, en tal sentido, Harrison, Birks, Franklin, y Mills (2017) mencionan, que los estudios de caso han experimentado un crecimiento metodológico importante en las últimas cuatro décadas, generándose un enfoque práctico y adaptable, que facilita el entendimiento de problemas de diversas especialidades. En lo que corresponde a la unidad de análisis, si bien muchos autores expresan la predilección de un caso único, es preciso señalar que se puede incrementar el alcance del estudio a una muestra de un grupo de casos, donde estos se vean afectados con el mismo problema. Para tal efecto, Creswell y Poth (2018) precisan que los estudios de caso a menudo involucran más de un caso. Por otro lado, respecto al problema de investigación que aborda el estudio, es indispensable tener en claro, el tipo de estudio de caso que se aplicará. En ese sentido, Creswell y Poth (2018) exponen que el objeto de aplicar un estudio de caso es 
analizar los procesos de casos muy particulares, es decir casos únicos e inusuales, que ameritan ser explicados de un modo muy minucioso, contando con la denominación de caso intrínseco. De forma simultánea, se presenta el estudio de caso colectivo, el cual tiene como finalidad entender un problema puntual, seleccionado para ello uno o más casos. Finalmente, una consideración fundamental que no se debe dejar de lado, es la naturaleza de origen disciplinario del estudio de caso, el cual parte de diversas disciplinas, ante ello Harrison, Birks, Franklin, y Mills (2017) declaran que el estudio de caso nace a partir de investigaciones de las especialidades de antropología, historia, psicología y sociología. Asimismo, los autores asignan sus raíces en investigaciones de antropología y ciencias sociales de inicios del siglo XX, donde se desarrollaron estudios etnográficos prolongados y minuciosos de personas y culturas.

Respecto a la aplicabilidad de los instrumentos cuantitativos en el método de estudio de caso, básicamente se refiere a la recolección de datos, lo cual es empleado solo como complemento del componente cualitativo del estudio, en especial, para la redacción en la información de resultados. En ese sentido, es preciso señalar que los investigadores de estudios de caso pueden recoger datos, tanto cuantitativos, como cualitativos (Goodyear, et al., 2016). Sin embargo, puede establecerse que la metodología de estudio de caso, podría ampliar sus aplicaciones, ya que está en permanente transformación y evolución, por ejemplo, los estudios de caso del tipo comparativo, son una herramienta para sintetizar la información o datos clave a lo largo del tiempo y espacio (Alpi y Evans, 2019).

Para evaluar la validez del método de estudio de caso, dentro de la investigación cualitativa, se considera la diversidad de los modelos con que cuenta, en ese sentido un diseño de estudio de caso integrado, involucra diferentes dimensiones de análisis, la riqueza del diseño determina el descubrimiento de la dinámica socio técnica de la adopción y sus implicaciones para la conexión social (Barbosa, Franz, Munteanu, y Baecker, 2017). En base a lo descrito, se establece que la aplicación del estudio de caso como investigación cualitativa, es más recurrente por parte de la comunidad científica, en especial a las disciplinas de ciencias sociales, medicina, psicología, antropología, entre otras.

En cuanto a la restricción relacionada a la muestra que se aplica en los estudios de caso, es decir en el empleo de un caso o casos, se puede compensar las limitaciones, verificándose el desarrollo de los procesos y los resultados con evidencia adicional, preferentemente con estudios diseñados de modo diferente, siendo recomendable utilizar metodologías mixtas para el desarrollo de futuras investigaciones ( $\mathrm{Zhu}$, Jia, y Lin, 2019). Asimismo, se enfatiza que, en la búsqueda de probar proposiciones generales, debe promoverse el descubrimiento de evidencia, que podría ser no ser compatible con los resultados cuantitativos (Moriarty, et al., 2019). En ese sentido, la idea central de tener un caso de muestra, no es solo probar la hipótesis de un solo caso, sino también demostrar la fecundidad de los métodos utilizados (Kivikkokangas y Leiman, 2019).

Entre las características del estudio de caso, que podrían considerarse como inconvenientes, es que son investigaciones básicamente subjetivas, dependiendo frecuentemente de la intervención y pericia del investigador, sumado a ello, las investigaciones por el método de caso pueden irse modificando gradualmente durante su desarrollo. Por ejemplo, si se investiga el comportamiento de un grupo de ancianos ante la presencia de las tecnologías de información, este grupo de estudio podría alterarse, una enfermedad, un cambio psicosomático o incluso la muerte, puede variar drásticamente la planeación de un estudio. Por 
otro lado, en base a que el método de caso es una investigación cualitativa, esta debe aplicarse por observación natural, sin que se establezca restricciones ante los comportamientos que sesguen la investigación. Del mismo modo, uno de los alcances del método de estudio de caso es la interpretación de las virtudes y alcance social de la investigación, aspectos que no pueden ser abordados por métodos con enfoque cuantitativo, para ello Barbosa, Franz, Munteanu, y Baecker (2017) precisan que, el método de estudio de caso permite descubrir la dinámica socio técnica de la adopción e implicaciones para la conexión social. En suma, el estudio de caso tiene limitaciones en comparación con los métodos cuantitativos, tal es el caso de Alpi y Evans (2019) que sugieren que, frente al enfoque de una investigación cuantitativa, los estudios por el método de caso tienen un alcance limitado y carecen de rigor científico, no siendo idóneos para generalizar o inferir los resultados obtenidos.

Cuando se establece contraindicaciones basadas en la ética de recopilación de información o metodología de trabajo, se debe sumar a ello, la rigurosidad de la investigación, para ello, Alpi y Evans (2019) sostienen que los estudios de caso representan maneras de explicar, describir o explorar fenómenos, sin embargo, desde una perspectiva cuantitativa, carecen de rigor y generalización. En ese sentido, es lógico considerar que tienen un enfoque limitado, refiriéndose a que es un estudio de una sola unidad, una persona o grupo de personas relacionadas, un evento o grupo de eventos, un documento o grupo de documentos relacionados (Moriarty et al., 2019). En oposición a lo señalado, se manifiesta que la investigación por el método de caso cumple con una serie de pasos que están dentro del marco del método científico, del mismo modo, para la obtención de la información recurre a fuentes confiables tales como, documentos de archivo de rigor científico, entrevistas, observación directa o con participación del investigador, así como el empleo de tecnologías de información. Del mismo modo, para una aplicación optima del método de caso, es necesaria una capacitación intensiva del investigador, el cual debe dominar el manejo de los instrumentos de recolección de datos, así como de análisis de información, ello le permitirá obtener resultados confiables que, si bien no se podrán inferir, permitirán alcanzar los objetivos de acuerdo a la investigación que se plantee. Asimismo, la experticia del investigador posibilitará la obtención de contribuciones importantes, desde un enfoque teórico y práctico, en tal sentido, representa un punto de partida para el desarrollo de futuras investigaciones. En suma, no se puede desmerecer el estudio de caso, ya que su aplicabilidad dependerá del problema abordado, para tal efecto Hernández y Mendoza (2018) postulan respecto a la validez de los enfoques de investigación, "Ninguna es intrínsecamente mejor que la otra, solo constituyen diferentes aproximaciones al estudio de un fenómeno" (p. 16).

\section{CONCLUSIONES}

Las ventajas del estudio de caso incluyen a la adaptabilidad, y la organización que proporciona para examinar diversos problemas, en ese sentido es un método eficiente para comenzar un estudio exploratorio. Por otro lado, son permisibles cuando se elige el perfil del caso e interpreta información a través diversos modos de conocimiento cualitativo y a través del papel del investigador durante el desarrollo del estudio.

Para el método de estudio de caso, la interpretación otorga significado a la información recopilada, considerando que la información sin analizar no tiene un significado intrínseco. Se precisa que los resultados de la aplicación del método de estudio de caso, deben ser tomados en cuenta como punto de inicio para el desarrollo de investigaciones posteriores, en poblaciones mayores 
con otros enfoques de estudio, como el cuantitativo o el mixto.

El método de estudio de caso contribuye de manera significativa en los aspectos teóricos y prácticos del conocimiento, en lo teórico incrementa las bases de la teoría existente, así como el conocimiento práctico con aportes en las ciencias sociales.

El método de estudio de caso, cuenta con limitaciones para muestras con muchos objetos de investigación, siendo aplicable para aquellas investigaciones donde se ha delimitado los objetivos.

Es de relevancia que, para el uso del método de estudio de caso se cuente con un investigador que domine los instrumentos de recolección de datos, así como del análisis de información, ello a fin de obtener resultados confiables que sean considerados como válidos por la comunidad científica.

El método de estudio de caso no prescinde del empleo de instrumentos cuantitativos, en ese sentido, los estudios cualitativos tendrían la capacidad de interpretar datos de investigaciones cuantitativas.

Se concluye, que ningún método es mejor que otro, siempre y cuando sea aplicado con la rigurosidad metodológica y con la validación de procedimientos e instrumentos de recolección de información y de desarrollo de análisis.

\section{REFERENCIAS}

Alpi, K., y Evans, J. (2019). Distinguishing Case Study as a Research Method from Case Reports as a Publication Type. Journal of the Medical Library Association, 1, 1-5

Avolio, B. (2015). Métodos Cualitativos De Investigación: Una Aplicación Al Estudio De Caso. México D.F.: Cengage

Barbosa, B., Franz, R., Munteanu, C., y Baecker, R. (2017). Adoption and Feasibility of a Communication App to Enhance Social
Connectedness amongst Frail Nstitutionalized Oldest Old: An Embedded Case Study. Information, Communication y Society, 21(11):1681-1699

Boydell, K., Hodgins, M., Gladstone, B., Stasiulis, E., Belliveau, G., y Cheu, H. (2016). Arts-Based Health Research and Academic Legitimacy: Transcending Hegemonic Conventions. Qualitative Research, 16(6):681-700

Bruns, W. (1989). Case Study Research: Design and Methods. Journal of Management Accounting Research, 1, 157-163

Creswell, J., y Poth, C. (2018). Qualitative Inquiry y Research Design: Choosing Among Five Approaches. Los Angeles: SAGE Publications

Cvitanovic, C., Cunningham, R., Dowd, A., Howden, S., y Putten, E. (2017). Using Social Network Analysis To Monitor And Assess The Effectiveness Of Knowledge Brokers At Connecting Scientists And Decision-Makers: An Australian Case Study. Environmental Policy and Governance, 27:256-269

Devaney, S., Spangler, A., Lee, A., y Delgadillo, L. (2018). Tips from the Experts on Conducting and Reviewing Qualitative Research. Family and Consumer Sciences Research Journal, 46(4): 396-405

Eisenhardt, K., Graebner, M., y Sonenshein, S. (2016). Grand Challenges and Inductive Methods: Rigor without Rigor Mortis. Academy Of Management Journal, 59(4):1113-1123

Flemming, K., Closs, S., Hughes, N., y Bennett, M. (2016). Using Qualitative Research to Overcome the Shortcomings of Systematic Reviews When Designing Of a Self-Management Intervention for Advanced Cancer Pain. International Journal of Qualitative Methods, 1-11

Goodyear, R., Borders, L., Chang, C., Guiffrida, D., Hutman, H., Kemer, G., y White, E. (2016). Prioritizing Questions and Methods for an International and Interdisciplinary Supervision Esearch Agenda: Suggestions by Eight Scholars. The Clinical Supervisor, 35(1):117-154 
Gupta, M., Sorlini, C., Linton, K., Duchen, R., y Lohfeld, L. (2016). The Hidden Ethics Curriculum in Two Canadian Psychiatry Residency Programs: A Qualitative Study. Accad Psychiatry, 40:592-599

Harrison, H., Birks, M., Franklin, R., y Mills, J. (2017). Case Study Research: Foundations and Methodological Orientations. In Forum Qualitative Sozialforschung, 18(1):1-17

Kivikkokangas, S., y Leiman, M. (2019). Amalia X: Dialogical Sequence Analysis as a Method in Theory-Based Single Case Research in Psychoanalysis. The Scandinavian Psychoanalytic Review, 41(2):108-118

Lee, J., y Catinga, S. (2017). What Do Geography Textbook Authors In England Consider When They Design Content And Select Case Studies? International Research in Geographical and Environmental Education, 26(4):342-356

Leonard, A., y Odutola, A. (2016). "Iamartistic": Mixed Method Case Study Research of Preservice Generalists' Perceptions of Arts in Education. Studies in Art Education, 57(3):279299

Morgenstern, R. (2018). Retrospective Analysis of U.S. Federal Environmental Regulation. Journal Benefit-Cost Analysis, 9(2):285-304

Moriarty, D., De Villavicencio, P., Black, L., Bustos, M., Cai, H., Mehlenbacher, B., y Mehlenbacher, A. (2019). Durable Research, Portable Findings: Rhetorical Methods in Case Study Research. Technical Communication Quarterly, 28(2):124136
Munn, Z., Peters, M., Stern, C., y Tufanaru, C. (2018). Systematic Review or Scoping Review? Guidance for Authors When Choosing Between a Systematic or Scoping Review Approach. BMC Medical Research Methodology, 18(143):1-7

Smith, P. (2018). Collecting Sufficient Evidence When Conducting a Case Study. The Qualitative Report, 23(5):1043-1048

Stake, R. (2005). Qualitative Case Studies. (Y. Lincoln, y N. Denzin, Eds.) Thousand Oaks, CA: Sage

Tetnowski, J. (2015). Qualitative Case Study Research Design. Perspectives on Fluency and Fluency Disorders, 25(1):39-45

Wyborn, C., Louder, E., Harrison, J., Montambault, J., Montana, J., Ryan, M., Reed, M. (2018). Understanding the impacts of research synthesis. Environmental Science and Policy, 86:72-84

Yin, R. (2014). Case Study Research: Design and Method (5th Ed.). Thousand Oaks, CA: Sage

Zhu, Q., Jia, R., y Lin, X. (2019). Building Sustainable Circular Agriculture in China: Economic Viability and Entrepreneurship. Management Decision, 57(4):1108-1122 